\title{
MATRIX INTERPRETATION OF MULTIPLE ORTHOGONALITY
}

\author{
A. BRANQUINHO, L. COTRIM, AND A. FOULQUIÉ MORENO
}

\begin{abstract}
In this work we give an interpretation of a $(s(d+1)+$ 1)-term recurrence relation in terms of type II multiple orthogonal polynomials. We rewrite this recurrence relation in matrix form and we obtain a three-term recurrence relation for vector polynomials with matrix coefficients. We present a matrix interpretation of the type II multi-orthogonality conditions. We state a Favard type theorem and the expression for the resolvent function associated to the vector of linear functionals. Finally a reinterpretation of the type II Hermite-Padé approximation in matrix form is given.
\end{abstract}

\section{INTRODUCTION}

Multiple orthogonal polynomials are a generalization of orthogonal polynomials in the sense that they satisfy orthogonality conditions with respect to a number of measures. Such polynomials arise, in a natural way, in the study of simultaneous rational approximation, and in particular for the study of Hermite-Padé approximation for a system of $d \in \mathbb{Z}_{+}$Markov functions (see [22]). In this way, multiple orthogonal polynomials are intimately related to Hermite-Padé approximation. In the literature we can find a lot of examples of multiple orthogonal polynomials (see $[1,2,3,13,17,19,24,25]$ ).

Let $\vec{n}=\left(n_{1}, \ldots, n_{d}\right) \in \mathbb{N}^{d}$ which is called a multi-index with length $|\vec{n}|:=n_{1}+\cdots+n_{d}$ and let $\left\{u^{1}, \ldots, u^{d}\right\}$ be a set of linear functionals $u^{j}: \mathbb{P} \rightarrow \mathbb{C}$ with $j=1,2, \ldots, d$.

Definition 1. Let $\left\{P_{\vec{n}}\right\}$ be a sequence of polynomials where the degree of $P_{\vec{n}}$ is at most $|\vec{n}|$. We say that $\left\{P_{\vec{n}}\right\}$ is a type II multiple orthogonal with respect to the set of linear functionals $\left\{u^{1}, \ldots, u^{d}\right\}$ and multi-index

Key words and phrases. Multiple-orthogonal polynomials and Hermite-Padé approximants and block tridiagonal operator and Favard type theorem.

2000 Mathemathics Subject Classification. Primary 33C45; Secondary 39B42.

The work of the first author was supported by CMUC/FCT. L. Cotrim thanks "Acção do Prodep" reference 5.3/C/00187.010/03 for their support. The work of A. Foulquié Moreno was support by UI Matemática e Aplicações from University of Aveiro. 
$\vec{n}=\left(n_{1}, \ldots, n_{d}\right) \in \mathbb{N}^{d}$, if

$$
u^{j}\left(x^{m} P_{\vec{n}}\right)=0, m=0,1, \ldots, n_{j}-1, \quad j=1, \ldots, d .
$$

For the particular case in which the set of linear functionals is a system of integrals with respect to positive Borel measures, $\mu_{j}$, on $I_{j} \subset \mathbb{R}, j=1, \ldots, d$, we have

$$
u^{j}\left(x^{k}\right)=\int_{I_{j}} x^{k} d \mu_{j}, \quad k \in \mathbb{N}, j=1, \ldots, d,
$$

and the conditions of multi-orthogonality, (1), can be rewritten as

$$
\int_{I_{j}} P_{\vec{n}}(x) x^{k} d \mu_{j}(x)=0, \quad k=0,1, \ldots, n_{j}-1, \quad j=1, \ldots, d .
$$

Definition 2. A multi-index $\vec{n}=\left(n_{1}, \ldots, n_{d}\right) \in \mathbb{N}^{d}$ is said to be normal for the set of linear functionals $\left\{u^{1}, \ldots, u^{d}\right\}$, if for any non trivial solution $P_{\vec{n}}$ of (1), the degree of $P_{\vec{n}}$ is equal to $|\vec{n}|$. When all the multi-indices of a given family are normal, we say that the set of linear functionals $\left\{u^{1}, \ldots, u^{d}\right\}$ is regular.

Definition 3. Let $u: \mathbb{P} \rightarrow \mathbb{C}$ be a linear functional, and $p \in \mathbb{P} a$ polynomial. The left product of $u$ by $p$, is the linear functional $p u$ : $\mathbb{P} \rightarrow \mathbb{C}$, defined by $p u\left(x^{j}\right)=u\left(p(x) x^{j}\right), j \in \mathbb{N}$.

In the works of K. Douak and P. Maroni [14], P. Maroni [20, 21], V. Kaliaguine [18], J. Van Iseghem [27], and also in the work of V.N. Sorokin and J. Van Iseghem [23], it can be seen that a sequence of type II multiple orthogonal polynomials with respect to the set of linear functionals $\left\{u^{1}, \ldots, u^{d}\right\}$ and multi-index $\vec{n}=\left(n_{1}, \ldots, n_{d}\right) \in \mathcal{J}$, where

$$
\begin{aligned}
\mathcal{J}=\{(0,0, \ldots, 0),(1,0, \ldots, 0), \ldots, & (1,1, \ldots, 1), \\
& (2,1, \ldots, 1), \ldots,(2,2, \ldots, 2), \ldots\},
\end{aligned}
$$

satisfy a $(d+2)$-term recurrence relation of type

$$
x B_{n}=B_{n+1}+\sum_{k=0}^{d} a_{n-k}^{n} B_{n-k}, a_{n-d}^{n} \neq 0, \text { for } n=d, \ldots .
$$

They call such polynomials $d$-orthogonal, where $d$ corresponds to the number of functionals.

Now, if we multiply this recurrence equation $s-1$ times by $x$ and using the recurrence relation property we arrive, for $n=s d, \ldots$, to

$$
x^{s} B_{n}=B_{n+s}+\sum_{k=0}^{s(d+1)-1} \tilde{a}_{n+s-1-k}^{n+s-1} B_{n+s-1-k}, \tilde{a}_{n-s d}^{n+s+1} \neq 0,
$$


which is our main object.

In this work we consider sequences of type II multiple orthogonal polynomials for more general sets of multi-indices, $\mathcal{J}$. We designate these multi-indices by quasi-diagonal of step $s$. In section 2 we build the sets of quasi-diagonal multi-indices $\mathcal{J}$. Next we give the type II multi-orthogonality conditions for a sequence of monic polynomials, $\left\{B_{n}\right\}$, i.e. $B_{n}=x^{n}+\cdots, n=0,1, \ldots$, with respect to the set of linear functionals $\left\{u^{1}, \ldots, u^{d}\right\}$ and a family of quasi-diagonal multi-indices $\mathcal{J}$ of step $s$. We also prove that this sequence satisfies a $(s(d+1)+1)$-term recurrence relation of the type (2). To finish this section, we rewrite the previous $(s(d+1)+1)$-term recurrence relation in matrix form and we obtain a three-term recurrence relation for vector polynomials with matrix coefficients. We also give an example of multiple Hermite orthogonal polynomials satisfying a three term vector recurrence relation with matrix coefficients. In section 3 we present an algebraic theory which enables us to operate with the new presented objects. Here, our main goal is to present a matrix interpretation in terms of a vector of functionals, of the multi-ortogonality conditions presented in the section 2 . We characterize the regularity (cf. $[6,12])$ of a set of linear functional in terms of the regularity of a vector of linear functionals. Next we give a result of existence and uniqueness of a type II sequence of vector orthogonal polynomials with respect to a regular vector of linear functionals $\mathcal{U}$, and using a matrix three-term recurrence relations we establish a Favard type theorem. We remark that other characterization for sequences of orthogonal polynomials in terms of matrix three-term recurrence relations can be found in $[15,16]$. In section 4 we express the resolvent function in terms of the matrix generating function associated to the vector of linear functionals. Note that in the recent paper [4], the authors applies the technique here exposed in the diagonal case, to describe the correspondence between dynamics of the coefficients of the operator defined by a Lax pair and its resolvent function. Finally, we give a reinterpretation of the type II multiple orthogonality, in terms of a Hermite-Padé approximation problem for the matrix generating function associated to the vector of linear functionals. We remark that Hermite-Padé approximation problems can be found for example in $[22,24]$, and in matrix form in $[5,7,8,9,10,11,26]$.

\section{QUASI-DiAgONAL MULTI-INDICES}

We call $\mathcal{J}$ a set of quasi-diagonal multi-indices of step $s$ if

$$
\mathcal{J}=\bigcup_{n \in \mathbb{N} \cup\{0\}} \mathcal{J}_{n}, \quad \mathcal{J}_{n}=\mathcal{J}_{0}+\{n(s, s, \ldots, s)\}, n \in \mathbb{N},
$$


and $\partial_{0}$ is called the pattern block and is explicitly given by

$$
\mathcal{J}_{0}=\left\{(0, \ldots, 0),(1,0, \ldots, 0), \ldots,\left(k_{i}^{1}, \ldots, k_{i}^{d}\right), \ldots,\left(k_{s d-1}^{1}, \ldots, k_{s d-1}^{d}\right)\right\},
$$

where $\left(k_{i}^{1}, \ldots, k_{i}^{d}\right)$, for $i=0, \ldots, s d-1$, verifies the following conditions:

a) $\left|\left(k_{i}^{1}, \ldots, k_{i}^{d}\right)\right|=i$,

b) $\left(k_{1}^{1}, \ldots, k_{1}^{d}\right)=(1,0, \ldots, 0)$,

c) $\left(k_{i}^{1}, \ldots, k_{i}^{d}\right) \leq\left(k_{i+1}^{1}, \ldots, k_{i+1}^{d}\right)$, i.e. increasing structure in each component,

d) $k_{i}^{j} \leq s$.

Notice that a) and c) implies that there exist a unique $j^{\prime}$ such that

$$
k_{i+1}^{j^{\prime}}=k_{i}^{j^{\prime}}+1 \text {, and } k_{i+1}^{j}=k_{i}^{j}, j \neq j^{\prime} .
$$

and $\mathrm{d})$ implies that $\left(k_{s d-1}^{1}, \ldots, k_{s d-1}^{d-1}, k_{s d-1}^{d}\right)$ is equal to $(s, \ldots, s, s-$ 1) up to a permutation. Remark that condition b) is not restrictive because we can always reorder the initial set of functionals.

For $s=1$ we have that $\mathcal{J}_{0}$ can be given by,

$$
\partial_{0}=\{(0, \ldots, 0),(1,0, \ldots, 0),(1,1, \ldots, 0), \ldots,(1, \ldots, 1,0)\},
$$

whose multi-indices we designate by diagonal.

There is an one-to-one correspondence, $\mathbf{i}$, between our set of quasidiagonal multi-indices $\mathcal{J} \subset \mathbb{N}^{d}$ and $\mathbb{N}$ given by, $\mathbf{i}(\vec{n})=|\vec{n}|=n$.

Let us consider, $B_{\vec{n}}$, a sequence of type II multiple orthogonal polynomial with respect to the set of linear functionals $\left\{u^{1}, \ldots, u^{d}\right\}$ and the set of quasi-diagonal multi-indices, $\mathcal{J}$. We identify $B_{\vec{n}} \equiv B_{|\vec{n}|}=B_{n}$, where $\vec{n}=\left(k_{n}^{1}, \ldots, k_{n}^{d}\right)$.

Algorithm (Construction of linear functionals). Let us consider a set of linear functionals $\left\{u^{1}, \ldots, u^{d}\right\}$ and a set of quasi-diagonal multiindices, $\mathcal{J}$, of step s.

Let $v^{1}=u^{1}, v^{i}=x^{k_{i-1}^{j}} u^{j}, i=2, \ldots$, sd where $j$, for each $i$, is uniquely defined by the condition $k_{i}^{j}=k_{i-1}^{j}+1$. Hence, we have

$$
v^{i} \in\left\{x^{k} u^{j}: k=0,1, \ldots, s-1, j=1,2, \ldots, d\right\}, i=1,2, \ldots, s d .
$$

Example 1. For the pattern block $\mathcal{J}_{0}=\{(0,0),(1,0),(2,0),(2,1),(2,2)$, $(3,2)\}$, we can obtain a new set of linear functionals, $\left\{v^{1}=u^{1}, v^{2}=\right.$ $\left.x u^{1}, v^{3}=u^{2}, v^{4}=x u^{2}, v^{5}=x^{2} u^{1}, v^{6}=x^{2} u^{2}\right\}$. Notice that we have used that $\left(k_{6}^{1}, k_{6}^{2}\right)=(3,3)$.

Theorem 1. The sequence of monic polynomials, $\left\{B_{n}\right\}$, is type II multiple orthogonal with respect to the regular set of linear functionals $\left\{u^{1}, \ldots, u^{d}\right\}$ and the set of quasi-diagonal multi-indices $\mathcal{J}$ of step $s$ if, 
and only if,

$$
\left\{\begin{array}{l}
v^{j}\left(\left(x^{s}\right)^{m} B_{s d r+i}\right)=0, m=0,1, \ldots, r-1, j=1, \ldots, s d \\
v^{\alpha}\left(\left(x^{s}\right)^{r} B_{s d r+i}\right)=0, \alpha=1, \ldots, i \\
v^{i+1}\left(\left(x^{s}\right)^{r} B_{s d r+i}\right) \neq 0,
\end{array}\right.
$$

where $i=0,1, \ldots, s d-1, r=0,1, \ldots$, and the linear functionals $v^{j}$, $j=1, \ldots$, sd are defined by the algorithm .

Proof. Let us consider the set of multi-indices

$$
\mathcal{J}_{0}=\left\{(0, \ldots, 0),(1,0, \ldots, 0), \ldots,\left(k_{i}^{1}, \ldots, k_{i}^{d}\right), \ldots,\left(k_{s d-1}^{1}, \ldots, k_{s d-1}^{d}\right)\right\} .
$$

The linear functionals $v^{1}, \ldots, v^{s d}$ are defined by the algorithm . We obtain the multi-orthogonality conditions for the polynomials $B_{i}, i=$ $1, \ldots, s d-1$. Let us consider the multi-index $\left(k_{i}^{1}, \ldots, k_{i}^{d}\right)$ and let $j \in$ $\{1, \ldots, d\}$ be uniquely defined by the condition $k_{i}^{j}=k_{i-1}^{j}+1$. We have

$$
u^{j}\left(x^{k_{i-1}^{j}} B_{i}\right)=0 \Leftrightarrow x^{k_{i-1}^{j}} u^{j}\left(B_{i}\right)=0 \Leftrightarrow v^{i}\left(B_{i}\right)=0 .
$$

By the increasing structure of the multi-indices, $B_{i}$ complies with the multi-orthogonality conditions of $B_{1}, \ldots, B_{i-1}$, so it holds,

$$
v^{j}\left(B_{i}\right)=0, \quad j=1, \ldots, i .
$$

We obtain the multi-orthogonality conditions for the polynomials $B_{r s d+i}, i=0,1, \ldots, s d-1, r=1, \ldots$

Let us consider the multi-index $\left(k_{i}^{1}, \ldots, k_{i}^{d}\right)+r(s, \ldots, s)$ and let $j \in$ $\{1, \ldots, d\}$ be uniquely defined by the condition $k_{i}^{j}=k_{i-1}^{j}+1$. We have $u^{j}\left(x^{k_{i-1}^{j}+r s} B_{r s d+i}\right)=0 \Leftrightarrow x^{k_{i-1}^{j}} u^{j}\left(\left(x^{s}\right)^{r} B_{r s d+i}\right)=0 \Leftrightarrow v^{i}\left(\left(x^{s}\right)^{r} B_{i+s d}\right)=0$.

By the increasing structure of the multi-indices, $B_{r s d+i}$ complies with the multi-orthogonality conditions of $B_{1}, \ldots, B_{r s d+i-1}$, so it holds that,

$$
\left\{\begin{array}{l}
v^{j}\left(\left(x^{s}\right)^{m} B_{s d r+i}\right)=0, m=0,1, \ldots, r-1, j=1, \ldots, s d \\
v^{\alpha}\left(\left(x^{s}\right)^{r} B_{s d r+i}\right)=0, \alpha=1, \ldots, i .
\end{array}\right.
$$

Finally, we show that $v^{i+1}\left(\left(x^{s}\right)^{r} B_{s d r+i}\right) \neq 0$, for $r=0, \ldots, s d-1$ and $i=0,1, \ldots$ In fact, if we suppose that $v^{1}\left(B_{0}\right)=0$, we get that $B_{1}$ is of degree 0 , which contradicts the normality of the multi-index $(1,0, \ldots, 0)$, and the third condition in (3) for $i=r=0$ is achieved. Now, let us suppose that,

$$
\left\{\begin{array}{l}
v^{j}\left(\left(x^{s}\right)^{m} B_{s d r+i}\right)=0, m=0,1, \ldots, r-1, j=1, \ldots, s d \\
v^{\alpha}\left(\left(x^{s}\right)^{r} B_{s d r+i}\right)=0, \alpha=1, \ldots, i \\
v^{i+1}\left(\left(x^{s}\right)^{r} B_{s d r+i}\right)=0 .
\end{array}\right.
$$


Then the polynomial $B_{s d r+i}$ satisfy the multi-orthogonality conditions of the polynomial $B_{s d r+i+1}$ which contradicts the normality of the multiindices. Hence, $v^{i+1}\left(\left(x^{s}\right)^{r} B_{s d r+i}\right) \neq 0$.

Reciprocally, for $n=s d r+i, i=1, \ldots, s d$

$$
\left\{\begin{array}{l}
v^{j}\left(\left(x^{s}\right)^{m} B_{s d r+i}\right)=0, m=0,1, \ldots, r-1, j=1, \ldots, s d \\
v^{\alpha}\left(\left(x^{s}\right)^{r} B_{s d r+i}\right)=0, \alpha=1, \ldots, i
\end{array}\right.
$$

and considering that the degree of $B_{n}$ is equal to $n$ by the normality of each of the multi-indices which implies the uniqueness of the monic type II multiple orthogonal polynomial sequence, $B_{n}$, with respect to the set of linear functionals $\left\{u^{1}, \ldots, u^{d}\right\}$ and quasi-diagonal multiindex $\vec{n}$ such that $|\vec{n}|=n$.

Here, we show that the sequence of monic type II multiple orthogonal polynomials, $\left\{B_{n}\right\}$, with respect to the regular set of linear functionals $\left\{u^{1}, \ldots, u^{d}\right\}$ and a set of quasi-diagonal multi-indices $\mathcal{J}$ of step $s$, satisfies a $(s(d+1)+1)$-term recurrence relation.

Theorem 2. Let $\left\{B_{n}\right\}$ be a monic type II multiple orthogonal polynomials sequence, with respect to a regular set of linear functionals $\left\{u^{1}, \ldots, u^{d}\right\}$ and a set of quasi-diagonal multi-indices $\mathcal{J}$ of step s. Then, there are sequences $\left(a_{n+s-1-k}^{n+s-1}\right) \subset \mathbb{C}, k=0,1, \ldots, s(d+1)-1$, such that,

$$
x^{s} B_{n}(x)=B_{n+s}(x)+\sum_{k=0}^{s(d+1)-1} a_{n+s-1-k}^{n+s-1} B_{n+s-1-k}(x),
$$

for $n=s d, s d+1, \ldots$, where $a_{n-s d}^{n+s-1} \neq 0$ and $B_{0}, B_{1}, \ldots, B_{s d-1}$ are given.

Proof. As the degree of the monic polynomials $B_{n}$ is equal to $n$, there is an unique sequence $\left(a_{j}^{n+s-1}\right) \subset \mathbb{C}$, such that:

$$
x^{s} B_{n}=B_{n+s}+\sum_{j=0}^{n+s-1} a_{j}^{n+s-1} B_{j} .
$$

Substituting $n$ by $s d r+i$ where $i=0,1, \ldots, s d-1$ and $r=0,1, \ldots$, in the above identity, we have

$$
x^{s} B_{s d r+i}-B_{s d r+i+s}=\sum_{j=0}^{s d r+i+s-1} a_{j}^{s d r+i+s-1} B_{j} .
$$

Now, considering the orthogonality conditions (3), and applying successively to both members of the above equation the functionals

$$
v^{1}, \ldots, v^{s d}, \ldots\left(x^{s}\right)^{r-2} v^{1}, \ldots,\left(x^{s}\right)^{r-2} v^{s d},\left(x^{s}\right)^{r-1} v^{1}, \ldots\left(x^{s}\right)^{r-1} v^{i}
$$


we obtain successively that $a_{j}^{s d r+i+s-1}=0$, for $j=0,1 \ldots,(r-1) s d+i$, and the theorem is proved.

Now we give a matrix interpretation for the recurrence relation(4).

Lemma 1. Let $\left\{B_{n}\right\}$ be a monic sequence of polynomials. Then, the following conditions are equivalent:

a) The sequence of polynomials $\left\{B_{n}\right\}$ satisfies a $(s(d+1)+1)$-term relation,

$x^{s} B_{n}(x)=B_{n+s}(x)+\sum_{k=0}^{s(d+1)-1} a_{n+s-1-k}^{n+s-1} B_{n+s-1-k}(x), n=s d, s d+1, \ldots$,

where $a_{n-s d}^{n+s-1} \neq 0$ and $B_{0}, B_{1}, \ldots, B_{s d-1}$ are given.

b) The vector sequence of polynomials $\left\{\mathcal{B}_{m}\right\}$, where

$$
\mathcal{B}_{m}=\left[\begin{array}{lll}
B_{m s d} & \cdots & B_{(m+1) s d-1}
\end{array}\right]^{T}, m \in \mathbb{N}
$$

satisfies a three-term recurrence relation with $s d \times$ sd matrix coefficients, for all $m=0,1, \ldots$,

$$
x^{s} \mathcal{B}_{m}(x)=\alpha_{m}^{s, d} \mathcal{B}_{m+1}(x)+\beta_{m}^{s, d} \mathcal{B}_{m}(x)+\gamma_{m}^{s, d} \mathcal{B}_{m-1}(x),
$$

with $\mathcal{B}_{-1}=0_{s d \times 1}$ and $\mathcal{B}_{0}$ given, where $\alpha_{m}^{s, d}, \beta_{m}^{s, d}$ and $\gamma_{m}^{s, d}$ are respectively given by

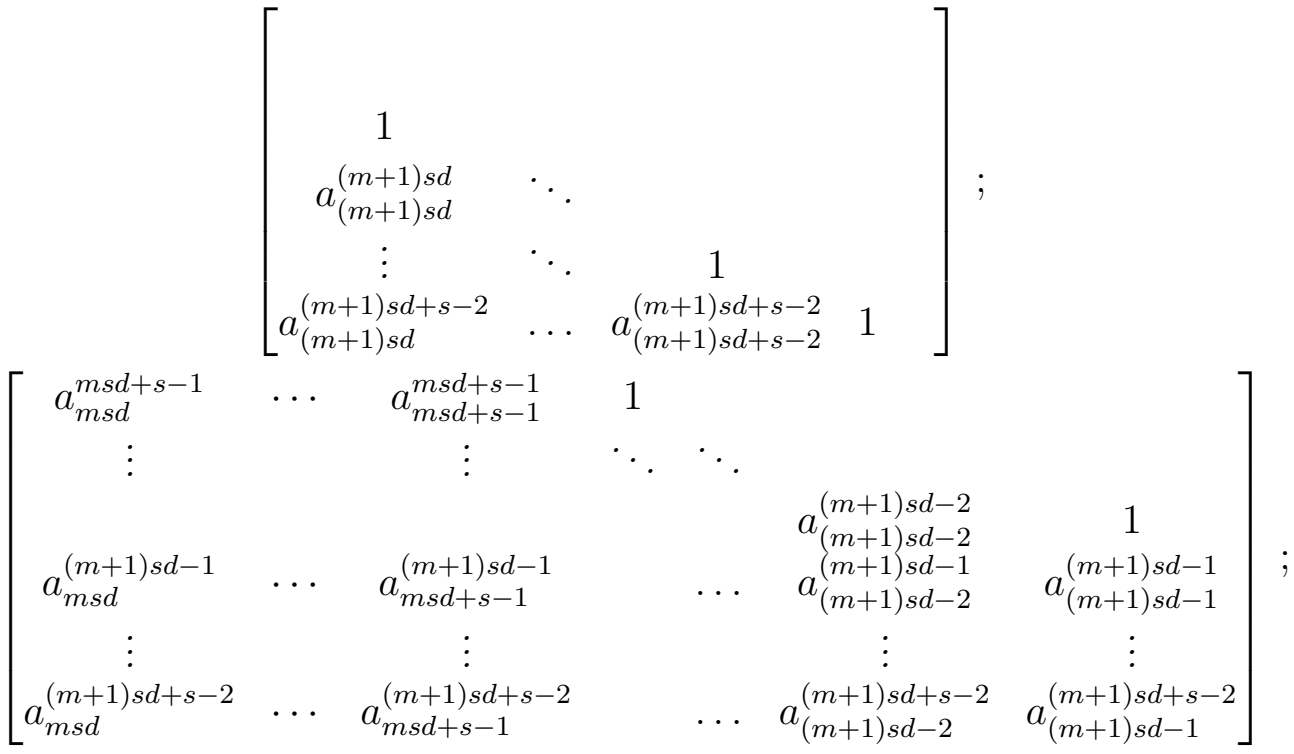

$$
\begin{aligned}
& {\left[\begin{array}{ccc}
a_{(m-1) s d}^{m s d+s-1} & \cdots & a_{m s d-1}^{m s d+s-1} \\
& \ddots & \vdots \\
& & a_{m s d-1}^{(m+1) s d+s-2}
\end{array}\right] \text {. }}
\end{aligned}
$$


Example 2. The Hermite multiple orthogonal polynomials for any multi-index $\left(n_{1}, n_{2}\right)$ are given by the Rodrigues formula (see [2])

$$
H_{\left(n_{1}, n_{2}\right)}(x)=\left(-\frac{1}{2}\right)^{n_{1}+n_{2}} e^{-b_{2} x+x^{2}} \frac{\partial^{n_{2}}}{\partial x^{n_{2}}}\left(e^{\left(-b_{1}+b_{2}\right) x} \frac{\partial^{n_{1}}}{\partial x^{n_{1}}}\left(e^{-b_{1} x+x^{2}}\right)\right)
$$

For the pattern block $\mathcal{J}_{0}=\{(0,0),(1,0),(2,0),(2,1)\}$ correspond to the case $s=d=2$ and the associated set of weight functions with support in $\mathbb{R}$ are given by $\left\{e^{-x^{2}+b_{1} x}, x e^{-x^{2}+b_{1} x}, e^{-x^{2}+b_{2} x}, x e^{-x^{2}+b_{2} x}\right\}$. Then, the three term recurrence relations with matrix coefficients $\alpha_{m}^{2,2}, \beta_{m}^{2,2}$ and $\gamma_{m}^{2,2}$

$$
x^{2} \mathcal{B}_{m}(x)=\alpha_{m}^{2,2} \mathcal{B}_{m+1}(x)+\beta_{m}^{2,2} \mathcal{B}_{m}(x)+\gamma_{m}^{2,2} \mathcal{B}_{m-1}(x), \quad m=0,1, \ldots,
$$

where

$$
\mathcal{B}_{m}(x)=\left[H_{(2 n, 2 n)}(x), H_{(2 n+1,2 n)}(x), H_{(2 n+2,2 n)}(x), H_{(2 n+2,2 n+1)}(x)\right]^{T}
$$

$\mathcal{B}_{-1}(x)=0_{4 \times 1}$, are given by

$$
\begin{aligned}
& {\left[\begin{array}{cccc}
0 & 0 & 0 & 0 \\
0 & 0 & 0 & 0 \\
1 & 0 & 0 & 0 \\
\left(b_{1}+b_{2}\right) 2 & 1 & 0 & 0
\end{array}\right],} \\
& {\left[\begin{array}{cccc}
\frac{2+b_{1}^{2}+16 n}{4} & b_{1} & & \\
\frac{b_{1}+2 b_{1} n+2 b_{2} n}{2} & \frac{6+b_{1}^{2}+16 n}{4} & 1 & 0 \\
\frac{1+6 n-b_{1} b_{2} n+b_{2}^{2} n+8 n^{2}}{2} & b_{1}+b_{1} n+b_{2} n & \frac{10+b_{2}^{2}+16 n}{4} & 1 \\
\frac{\left(b_{1}-b_{2}\right)(1+2 n)}{4} & \frac{3+b_{1}^{2}-b_{1} b_{2}+10 n+b_{1}^{2} n-b_{1} b_{2} n+8 n^{2}}{2} & \frac{2 b_{1}+b_{2}+2 b_{1} n+2 b_{2} n}{2} & \frac{14+b_{2}^{2}+16 n}{4}
\end{array}\right],} \\
& {\left[\begin{array}{cccc}
\frac{\left(b_{1}-b_{2}\right)^{2} n(-1+2 n)}{8} & \frac{\left(b_{1}-b_{2}\right) n\left(-2+b_{1}^{2}-b_{1} b_{2}+8 n\right)}{4} & \frac{n\left(-2+b_{1}^{2}-b_{1} b_{2}+8 n\right)}{2} & \left(b_{1}+b_{2}\right) n \\
0 & \frac{\left(b_{1}-b_{2}\right)^{2} n(1+2 n)}{8} & \frac{\left(b_{1}-b_{2}\right) n}{2} & \frac{n\left(2-b_{1} b_{2}+b_{2}^{2}+8 n\right)}{2} \\
0 & 0 & \frac{\left(b_{1}-b_{2}\right)^{2}(-1+2 n)}{8} & \frac{-\left(\left(b_{1}-b_{2}\right) n\left(2-b_{1} b_{2}+b_{2}^{2}+8 n\right)\right)}{4} \\
0 & 0 & 0 & \frac{\left(b_{1}-b_{2}\right)^{2} n(1+2 n)}{8}
\end{array}\right] .}
\end{aligned}
$$

3. Matrix interpretation of TYPE II MUlti-ORThOGONALity

Let us consider

$$
\mathbb{P}^{s d}=\left\{\left[\begin{array}{lll}
P_{1} & \cdots & P_{s d}
\end{array}\right]^{T}: P_{j} \in \mathbb{P}\right\} .
$$

We denote by $\mathcal{M}_{s d \times s d}$ the set of $s d \times s d$ matrices with entries in $\mathbb{C}$.

Let $\left\{\mathcal{P}_{j}\right\}$ be a sequence of vectors of polynomials given by

$$
\mathcal{P}_{j}=\left[\begin{array}{lll}
x^{j s d} & \cdots & x^{(j+1) s d-1}
\end{array}\right]^{T}, j \in \mathbb{N} .
$$


Definition 4. Let $v^{j}: \mathbb{P} \rightarrow \mathbb{C}$ with $j=1, \ldots$, sd be linear functionals. We define the vector of functionals $\mathcal{U}=\left[\begin{array}{lll}v^{1} & \cdots & v^{\text {sd }}\end{array}\right]^{T}$ acting in $\mathbb{P}^{s d}$ over $\mathcal{M}_{s d \times s d}$, by

$$
\mathcal{U}(\mathcal{P}):=\left(\mathcal{U} \cdot \mathcal{P}^{T}\right)^{T}=\left[\begin{array}{ccc}
v^{1}\left(P_{1}\right) & \cdots & v^{s d}\left(P_{1}\right) \\
\vdots & \ddots & \vdots \\
v^{1}\left(P_{s d}\right) & \cdots & v^{s d}\left(P_{s d}\right)
\end{array}\right]
$$

where "" means the symbolic product of the vectors $\mathcal{U}$ and $\mathcal{P}^{T}$.

Now we define an operation called left multiplication of a vector of functionals by a polynomial.

Definition 5. Let $\widehat{A}=\sum_{k=0}^{l} A_{k} x^{k}$ be a matrix polynomial of degree $l$ where $A_{k} \in \mathcal{M}_{s d \times s d}$ and $\mathcal{U}$ a vector of linear functionals. We define the vector of linear functionals, left multiplication of $\mathcal{U}$ by a matrix polynomial $\widehat{A}$, and denote it by $\widehat{A} \mathcal{U}$, the map of $\mathbb{P}^{s d}$ to $\mathcal{M}_{s d \times s d}$, defined by:

$$
(\widehat{A} \mathcal{U})(\mathcal{P}):=\left(\widehat{A} \mathcal{U} \cdot \mathcal{P}^{T}\right)^{T}=\sum_{k=0}^{l}\left(x^{k} \mathcal{U}\right)(\mathcal{P})\left(A_{k}\right)^{T} .
$$

Theorem 3. A sequence of monic polynomials $\left\{B_{m}\right\}$, is type II multiple orthogonal with respect to the regular set of linear functionals $\left\{u^{1}, \ldots, u^{d}\right\}$ and the set of quasi-diagonal multi-indices $\mathcal{J}$ of step $s$ if, and only if, the vector sequence of polynomials $\left\{\mathcal{B}_{m}\right\}, \mathcal{B}_{m}=\left[B_{m s d} \cdots\right.$ $\left.B_{(m+1) s d-1}\right]^{T}, m \in \mathbb{N}$, satisfies:

$$
\left.\begin{array}{l}
\text { i) }\left(\left(x^{s}\right)^{k} \mathcal{U}\right)\left(\mathcal{B}_{m}\right)=0_{s d \times s d}, k=0,1, \ldots, m-1 \\
\text { ii) }\left(\left(x^{s}\right)^{m} \mathfrak{U}\right)\left(\mathcal{B}_{m}\right)=\Delta_{m},
\end{array}\right\}
$$

where $\mathcal{U}=\left[\begin{array}{lll}v^{1} & \ldots & v^{s d}\end{array}\right]^{T}, v^{j}, j=1, \ldots$, sd are defined by the algorithm, and $\Delta_{m}$ is a regular upper triangular sd $\times$ sd matrix.

Proof. By Definition 4, we have

$$
\left(\left(x^{s}\right)^{k} \mathcal{U}\right)\left(\mathcal{B}_{m}\right)=\left[\begin{array}{ccc}
v^{1}\left(\left(x^{s}\right)^{k} B_{m s d}\right) & \cdots & v^{s d}\left(\left(x^{s}\right)^{k} B_{m s d}\right) \\
\vdots & \ddots & \vdots \\
v^{1}\left(\left(x^{s}\right)^{k} B_{(m+1) s d-1}\right) & \cdots & v^{s d}\left(\left(x^{s}\right)^{k} B_{(m+1) s d-1}\right)
\end{array}\right]
$$

Using the orthogonality conditions of type II in Theorem 1 we have the conditions (7), and reciprocally.

Now we introduce the notions of moments and Hankel matrices by blocks associated to the vector of linear functionals $\mathcal{U}=\left[\begin{array}{lll}v^{1} & \cdots & v^{s d}\end{array}\right]^{T}$. 
Definition 6. We define the moments of order $j \in \mathbb{N}$ associated to the vector of linear functionals $\left(x^{s}\right)^{k} \mathcal{U}$, by

(8) $\mathcal{U}_{j}^{k}=\left(\left(x^{s}\right)^{k} \mathcal{U}\right)\left(\mathcal{P}_{j}\right)=\left[\begin{array}{ccc}v^{1}\left(x^{j s d+k s}\right) & \cdots & v^{s d}\left(x^{j s d+k s}\right) \\ \vdots & \ddots & \vdots \\ v^{1}\left(x^{(j+1) s d+k s-1}\right) & \cdots & v^{s d}\left(x^{(j+1) s d+k s-1}\right)\end{array}\right]$

we define Hankel matrices by

$$
\mathcal{H}_{m}=\left[\begin{array}{ccc}
\mathcal{U}_{0}^{0} & \cdots & \mathcal{U}_{0}^{m} \\
\vdots & \ddots & \vdots \\
\mathcal{U}_{m}^{0} & \cdots & \mathcal{U}_{m}^{m}
\end{array}\right], m \in \mathbb{N}
$$

and we say that the vector of linear functionals $\mathcal{U}$ is regular if the principal minors of the matrix $\mathcal{H}_{m}$, are regular for $m \in \mathbb{N}$.

If we consider the matrix $A=\left(a_{i, j}\right)$, we denote by $A_{r, s}, r, s \in \mathbb{N}$ the principal submatrix obtained from $A$ of size $r \times s$, that is $A_{r, s}=$ $\left(a_{i, j}\right), i=1, \ldots, r, j=1, \ldots, s$.

Theorem 4. Let us consider a set of linear functionals $\left\{u^{1}, \ldots, u^{d}\right\}$ and the set of quasi-diagonal multi-indices $\mathcal{J}$ of step $s$ and let $\mathcal{U}=$ $\left[v^{1} \ldots v^{s d}\right]^{T}$ be the vector of linear functionals where $v^{j}, j=1, \ldots, s d$ are defined by the algorithm. Then $\mathcal{U}$ is regular if, and only if, $\left\{u^{1}, \ldots\right.$, $\left.u^{d}\right\}$ is regular.

Proof. Let us suppose that the set of linear functionals $\left\{u^{1}, \ldots, u^{d}\right\}$ is regular. Let $\left\{B_{n}\right\}$ be the sequence of monic polynomials multiple orthogonal with respect to this set of linear functionals and the set of quasi-diagonal multi-indices $\mathcal{J}$, where $\operatorname{deg} B_{n}=n$. Let us consider the vector sequence of polynomials associated $\left\{\mathcal{B}_{m}\right\}$, given by $\mathcal{B}_{m}=$ $\left[\begin{array}{lll}B_{m s d} & \cdots & B_{(m+1) s d-1}\end{array}\right]^{T}, m \in \mathbb{N}$. We can write $\mathcal{B}_{m}=\sum_{j=0}^{m} B_{j}^{m} \mathcal{P}_{j}$ where $B_{j}^{m} \in \mathcal{M}_{s d \times s d}$. and $B_{m}^{m}$ a regular lower triangular matrix. By the multi-orthogonality conditions (7) the vector sequence of polynomials $\left\{\mathcal{B}_{m}\right\}$ satisfies

$$
\left(\left(x^{s}\right)^{k} \mathcal{U}\right)\left(\mathcal{B}_{m}\right)=\left(\left(x^{s}\right)^{k} \mathcal{U}\right)\left(\sum_{j=0}^{m} B_{j}^{m} \mathcal{P}_{j}\right)=\sum_{j=0}^{m} B_{j}^{m}\left(\left(x^{s}\right)^{k} \mathcal{U}\right)\left(\mathcal{P}_{j}\right)=0_{s d \times s d}
$$

and for all $m \in \mathbb{N}$,

$$
\left(\left(x^{s}\right)^{m} \mathcal{U}\right)\left(\mathcal{B}_{m}\right)=\sum_{j=0}^{m} B_{j}^{m}\left(\left(x^{s}\right)^{m} \mathcal{U}\right)\left(\mathcal{P}_{j}\right)=\Delta_{m}
$$


where $\Delta_{m}$ are regular upper triangular matrices of order $s d \times s d$. In matrix form we have,

$$
\left[\begin{array}{lll}
B_{0}^{m} & \cdots & B_{m}^{m}
\end{array}\right]\left[\begin{array}{ccc}
\mathcal{U}_{0}^{0} & \cdots & \mathcal{U}_{0}^{m} \\
\vdots & \ddots & \vdots \\
\mathcal{U}_{m}^{0} & \cdots & \mathcal{U}_{m}^{m}
\end{array}\right]=\left[\begin{array}{lllll}
0_{s d \times s d} & \cdots & 0_{s d \times s d} & \Delta_{m}
\end{array}\right] .
$$

For $m=0$, we have $B_{0}^{0} \mathcal{U}_{0}^{0}=\Delta_{0}$. Using the regularity of the principal minors of the matrices $\mathcal{B}_{0}^{0}$ and $\Delta_{0}$ we have that the principal minors of $U_{0}^{0}$ are regular matrices.

For $m=1$ we have

$$
\left\{\begin{array}{l}
B_{0}^{1} \mathfrak{U}_{0}^{0}+B_{1}^{1} \mathfrak{U}_{1}^{0}=0_{s d \times s d} \\
B_{0}^{1} \mathfrak{U}_{0}^{1}+B_{1}^{1} \mathfrak{U}_{1}^{1}=\Delta_{1},
\end{array} \quad \text { i.e. } \quad B_{1}^{1}\left(\mathfrak{U}_{1}^{1}-\mathfrak{U}_{1}^{0}\left(\mathfrak{U}_{0}^{0}\right)^{-1} \mathfrak{U}_{0}^{1}\right)=\Delta_{1} .\right.
$$

Using the regularity of the principal minors of the matrices $\mathcal{B}_{1}^{1}$ and $\Delta_{1}$ we have that the principal minors of $\left(\mathcal{U}_{1}^{1}-\mathcal{U}_{1}^{0}\left(\mathcal{U}_{0}^{0}\right)^{-1} \mathcal{U}_{0}^{1}\right)$ are regular matrices, and using the triangular structure by blocks we have that the principal minors of $\mathcal{H}_{1}$ are regular matrices. This argument can be inductively repeated and we obtain the regularity of $\mathcal{U}$.

Reciprocally, supposing the regularity of the vector of linear functionals $\mathcal{U}$, let us consider $B_{n}(x)=a_{n, n} x^{n}+a_{n, n-1} x^{n-1}+\cdots+a_{n, 0}$. The multiorthogonal conditions for the multi-index $\vec{n}$, such that $|\vec{n}|=m s d+i$, for $m \in \mathbb{N}, 0 \leq i \leq s d-1$, are:

$$
\left[\begin{array}{lll}
a_{m s d+i, 0} & \cdots & a_{m s d+i, m s d+i}
\end{array}\right]\left[\begin{array}{ccc}
\mathcal{U}_{0}^{0} & \cdots & \mathcal{U}_{0}^{m} \\
\vdots & \ddots & \vdots \\
\mathcal{U}_{m}^{0} & \cdots & \mathcal{U}_{m}^{m}
\end{array}\right]_{m s d+i+1, m s d+i}=0_{1 \times(m s d+i)}
$$

Let us suppose that there exist a solution for this linear system of equation of degree less than $m s d+i$, that is $a_{m s d+i, m s d+i}=0$. In this case we would have a linear homogeneous system of $m s d+i$ equations and unknowns and the matrix of coefficients being a regular matrix. This would imply that the unique solution is the trivial solution and so the multi-index $\vec{n}$ such that $|\vec{n}|=m s d+i$ is a normal index.

Theorem 5. Let $\mathcal{U}=\left[\begin{array}{lll}v^{1} & \cdots & v^{s d}\end{array}\right]^{T}$ be a vector of linear functionals. Then $\mathcal{U}=\left[\begin{array}{lll}v^{1} & \cdots & v^{\text {sd }}\end{array}\right]^{T}$ is regular if, and only if, there exist a unique vector sequence of polynomials $\left\{\mathcal{B}_{m}\right\}, \mathcal{B}_{m}=\left[\begin{array}{lll}B_{m s d} & \cdots & B_{(m+1) s d-1}\end{array}\right]$, where $B_{n}$ is a monic polynomial of degree $n$ and a unique sequence $\Delta_{m}, m \in \mathbb{N}$, of regular upper triangular $s d \times$ sd matrices such that:

$$
\left(\left(x^{s}\right)^{k} \mathcal{U}\right)\left(\mathcal{B}_{m}\right)=\Delta_{m} \delta_{k, m}, \quad k=0,1, \ldots, m, \quad m \in \mathbb{N},
$$


Proof. Let us suppose that $\mathcal{U}$ is regular. To find the vector polynomial sequence $\left\{\mathcal{B}_{m}\right\}$, where $\mathcal{B}_{m}=\left[\begin{array}{lll}B_{m s d} & \cdots & B_{(m+1) s d-1}\end{array}\right]^{T}, m \in \mathbb{N}$, and $B_{n}$ a monic polynomial of degree $n$, that satisfies

$$
\left(\left(x^{s}\right)^{k} \mathcal{U}\right)\left(\mathcal{B}_{m}\right)=\Delta_{m} \delta_{k, m}, \quad k=0,1, \ldots, m, \quad m \in \mathbb{N},
$$

is equivalent to solve

$$
\left[\begin{array}{lll}
B_{0}^{m} & \cdots & B_{m}^{m}
\end{array}\right]\left[\begin{array}{ccc}
\mathcal{U}_{0}^{0} & \cdots & \mathcal{U}_{0}^{m} \\
\vdots & \ddots & \vdots \\
\mathcal{U}_{m}^{0} & \cdots & \mathcal{U}_{m}^{m}
\end{array}\right]=\left[\begin{array}{lllll}
0_{s d \times s d} & \cdots & 0_{s d \times s d} & \Delta_{m}
\end{array}\right]
$$

where we write $\mathcal{B}_{m}=\sum_{j=0}^{m} B_{j}^{m} \mathcal{P}_{j}$, with $B_{j}^{m} \in \mathcal{M}_{s d \times s d}$. and $B_{m}^{m}$ a regular lower triangular matrix.

For $m=0$, we have $B_{0}^{0} \mathcal{U}_{0}^{0}=\Delta_{0}$.

Using the regularity of the principal minors of the matrices $\mathcal{U}_{0}^{0}$, and the $L U$ decomposition, we can find uniquely $B_{0}^{0}$ a regular lower triangular matrix with entries equal to 1 in the main diagonal, and $\Delta_{0}$ a regular upper triangular matrix such that $B_{0}^{0} \mathfrak{U}_{0}^{0}=\Delta_{0}$.

For $m=1$ we have

$$
\left\{\begin{array}{l}
B_{0}^{1} \mathcal{U}_{0}^{0}+B_{1}^{1} \mathfrak{U}_{1}^{0}=0_{s d \times s d} \\
B_{0}^{1} \mathcal{U}_{0}^{1}+B_{1}^{1} \mathfrak{U}_{1}^{1}=\Delta_{1},
\end{array} \quad \text { i.e. } B_{1}^{1}\left(\mathcal{U}_{1}^{1}-\mathcal{U}_{1}^{0}\left(\mathcal{U}_{0}^{0}\right)^{-1} \mathfrak{U}_{0}^{1}\right)=\Delta_{1} .\right.
$$

Again using the regularity of the principal minors of the matrices $\mathcal{U}_{1}^{1}$ $-\mathcal{U}_{1}^{0}\left(\mathcal{U}_{0}^{0}\right)^{-1} \mathcal{U}_{0}^{1}$, and the $L U$ decomposition, we can find uniquely $B_{1}^{1}$ a regular lower triangular matrix with entries equal to 1 in the main diagonal, and $\Delta_{1}$ a regular upper triangular matrix such that $B_{1}^{1}\left(\mathcal{U}_{1}^{1}-\right.$ $\left.\mathcal{U}_{1}^{0}\left(\mathcal{U}_{0}^{0}\right)^{-1} \mathcal{U}_{0}^{1}\right)=\Delta_{1}$. We also obtain from $B_{0}^{1} \mathcal{U}_{0}^{0}+B_{1}^{1} \mathcal{U}_{1}^{0}=0_{s d \times s d}$, uniquely the matrix $B_{0}^{1}$. This argument can be inductively repeated and we obtain the stated result.

The converse is true following the same reasoning as in Theorem 4.

In section 2 we have proven that a sequence of monic type II multiple orthogonal polynomials, $\left\{B_{n}\right\}$, with respect to the regular set of linear functionals $\left\{u^{1}, \ldots, u^{d}\right\}$ and the set of quasi-diagonal multi-indices $\mathcal{J}$ of step $s$ satisfy a $(s(d+1)+1)$-term recurrence relation and we rewrote this recurrence relation in matrix form, obtaining a three-term recurrence relation for vector polynomials with matrix coefficients. Now we prove the converse of this result which is called the Favard type theorem. Note that in the given literature (see for instance $[15,16]$ ), the coefficients of matrix three term recurrence relation, are regular Hermitian matrices, and in (5) this is not the case.

Theorem 6. Let $\left\{B_{n}\right\}$ be a sequence of monic polynomials and let us consider the vector sequence of polynomials associated $\left\{\mathcal{B}_{m}\right\}, \mathcal{B}_{m}=$ 
$\left[\begin{array}{lll}B_{m s d} & \cdots & B_{(m+1) s d-1}\end{array}\right]$ and let $\mathcal{U}=\left[\begin{array}{lll}v^{1} & \cdots & v^{s d}\end{array}\right]^{T}$ be a vector of linear functionals. Then, the following conditions are equivalent:

a) The vector sequence of polynomials $\left\{\mathcal{B}_{m}\right\}$ satisfies:

$$
\left(\left(x^{s}\right)^{k} \mathcal{U}\right)\left(\mathcal{B}_{m}\right)=\Delta_{m} \delta_{k, m}, \quad k=0,1, \ldots, m, \quad m \in \mathbb{N},
$$

where $\Delta_{m}$ is a regular upper triangular $s d \times s d$ matrix.

b) There exist sequences of $s d \times s d$ matrices $\left(\alpha_{m}^{s, d}\right),\left(\beta_{m}^{s, d}\right)$ and $\left(\gamma_{m}^{s, d}\right)$, $m \in \mathbb{N}$, with $\gamma_{m}^{s, d}$ regular upper triangular matrix such that $\mathcal{B}_{m}$ is defined by the three-term recurrence relation with matrix coefficients in $\mathcal{M}_{s d \times s d}$ given for all $m=0,1, \ldots$ by

$$
x^{s} \mathcal{B}_{m}(x)=\alpha_{m}^{s, d} \mathcal{B}_{m+1}(x)+\beta_{m}^{s, d} \mathcal{B}_{m}(x)+\gamma_{m}^{s, d} \mathcal{B}_{m-1}(x),
$$

with $\mathcal{B}_{-1}=0_{d \times 1}$ and $\mathcal{B}_{0}$ given.

Furthermore

$$
\Delta_{m}=\gamma_{m}^{s, d} \cdots \gamma_{1}^{s, d} \Delta_{0}, \quad m=1,2, \ldots
$$

Proof. $a) \Rightarrow b$ ). We can express:

$$
\begin{aligned}
x^{s} \mathcal{B}_{m}(x)=\alpha_{m}^{s, d} \mathcal{B}_{m+1}(x)+\beta_{m}^{s, d} \mathcal{B}_{m}(x) & +\gamma_{m}^{s, d} \mathcal{B}_{m-1}(x) \\
& +\sum_{j=0}^{m-2} \delta_{m, j}^{s, d} \mathcal{B}_{j}(x), m=0,1, \ldots
\end{aligned}
$$

where $\alpha_{m}^{s, d}, \beta_{m}^{s, d}, \gamma_{m}^{s, d}, \delta_{m, j}^{s, d} \in \mathcal{M}_{s d \times s d}$ and they are uniquely determined. For $m \geq 2$, let us multiply both members of this equation by $\left(x^{s}\right)^{k}$,

$$
\begin{aligned}
\left(x^{s}\right)^{(k+1)} \mathcal{B}_{m}(x)=\alpha_{m}^{s, d}\left(x^{s}\right)^{k} & \mathcal{B}_{m+1}(x)+\beta_{m}^{s, d}\left(x^{s}\right)^{k} \mathcal{B}_{m}(x) \\
& +\gamma_{m}^{s, d}\left(x^{s}\right)^{k} \mathcal{B}_{m-1}(x)+\sum_{j=0}^{m-2} \delta_{m, j}^{s, d}\left(x^{s}\right)^{k} \mathcal{B}_{j}(x) .
\end{aligned}
$$

For $k=0, \ldots, m-2$ we apply successively the vector of functionals $\mathcal{U}$, use the linearity, the orthogonality condition (11), and we obtain

$$
0_{s d \times s d}=\delta_{m, j}^{s, d} \Delta_{j}, \quad j=0, \ldots, m-2 ;
$$

from the regularity of the matrix $\Delta_{j}$, for $j=0, \ldots, m-2$ we get that

$$
0_{s d \times s d}=\delta_{m, m-1}^{s, d}, \quad j=0, \ldots, m-2 .
$$

For $k=m-1$ we obtain $\Delta_{m}=\gamma_{m}^{s, d} \Delta_{m-1}$ so it holds that $\gamma_{m}^{s, d}$ is a regular upper triangular matrix.

$b) \Rightarrow a)$. We build a vector of linear functionals $\mathcal{U}$ that verifies $(11)$ defined uniquely taking into account its moments $\mathcal{U}_{m}^{k}$. For each $m \in$ $\mathbb{N}$, there is an unique sequence $\left(B_{j}^{m}\right) \subset \mathcal{M}_{s d \times s d}$, such that, $\mathcal{B}_{m}=$ 
$\sum_{j=0}^{m} B_{j}^{m} \mathcal{P}_{j}$.

- Let $k=0$. We have

$$
\mathcal{U}\left(\mathcal{B}_{0}\right)=B_{0}^{0} \mathfrak{U}\left(\mathcal{P}_{0}\right)
$$

and so $\mathcal{U}_{0}^{0}=\left(B_{0}^{0}\right)^{-1} \mathcal{U}\left(\mathcal{B}_{0}\right)$,

$\mathcal{U}\left(\mathcal{B}_{m}\right)=\sum_{j=0}^{m} B_{j}^{m} \mathcal{U}\left(\mathcal{P}_{j}\right)$, i.e. $\mathcal{U}_{m}^{0}=-\sum_{j=0}^{m-1}\left(B_{m}^{m}\right)^{-1} B_{j}^{m} \mathcal{U}_{j}^{0}, m=1,2, \ldots$

- Let $k=1,2, \ldots$ Using (12) we have

$$
\left(x^{s}\right)^{k} \mathcal{B}_{m}=\alpha_{m}^{s, d} x^{s(k-1)} \mathcal{B}_{m+1}+\beta_{m}^{s, d} x^{s(k-1)} \mathcal{B}_{m}+\gamma_{m}^{s, d} x^{s(k-1)} \mathcal{B}_{m-1} .
$$

For $m=0$ we have

$$
\mathcal{U}\left(\left(x^{s}\right)^{k} \mathcal{B}_{0}\right)=\alpha_{0}^{s, d} \mathcal{U}\left(x^{s(k-1)} \mathcal{B}_{1}\right)+\beta_{0}^{s, d} \mathcal{U}\left(x^{s(k-1)} \mathcal{B}_{0}\right),
$$

i.e. $\mathfrak{U}_{0}^{k}=\left(B_{0}^{0}\right)^{-1}\left[\alpha_{0}^{s, d} B_{1}^{1} \mathfrak{u}_{1}^{s(k-1)}+\left(\alpha_{0}^{s, d} B_{0}^{1}+\beta_{0}^{s, d} B_{0}^{0}\right)\right] \mathfrak{U}_{0}^{s(k-1)}$.

For $m \leq k$, we have

$$
\begin{aligned}
& \mathcal{U}\left(\left(x^{s}\right)^{k} \mathcal{B}_{m}\right)=\alpha_{m}^{s, d} \mathcal{U}\left(x^{s(k-1)} \mathcal{B}_{m+1}\right)+\beta_{m}^{s, d} \mathcal{U}\left(x^{s(k-1)} \mathcal{B}_{m}\right) \\
& +\gamma_{m}^{s, d} \mathcal{U}\left(x^{s(k-1)} \mathcal{B}_{m-1}\right), \\
& \mathcal{U}\left(\left(x^{s}\right)^{k} \mathcal{B}_{m}\right)=\alpha_{m}^{s, d} \sum_{j=0}^{m+1} B_{j}^{m+1} \mathcal{U}_{j}^{k-1}+\beta_{m}^{s, d} \sum_{j=0}^{m} B_{j}^{m} \mathfrak{u}_{j}^{k-1} \\
& +\gamma_{m}^{s, d} \sum_{j=0}^{m-1} B_{j}^{m-1} \mathcal{u}_{j}^{k-1} \\
& \mathcal{U}\left(\left(x^{s}\right)^{k} \mathcal{B}_{m}\right)=\sum_{j=0}^{m-1}\left(\alpha_{m}^{s, d} B_{j}^{m+1}+\beta_{m}^{s, d} B_{j}^{m}+\gamma_{m}^{s, d} B_{j}^{m-1}\right) \mathcal{U}_{j}^{k-1} \\
& +\left(\alpha_{m}^{s, d} B_{m}^{m+1}+\beta_{m}^{s, d} B_{m}^{m}\right) \mathcal{U}_{m}^{k-1}+\alpha_{m}^{s, d} B_{m+1}^{m+1} \mathcal{U}_{m+1}^{k-1} .
\end{aligned}
$$

Taking into account that,

$$
\mathcal{U}\left(\left(x^{s}\right)^{k} \mathcal{B}_{m}\right)=\mathcal{U}\left(\left(x^{s}\right)^{k} \sum_{j=0}^{m} B_{j}^{m} \mathcal{P}_{j}\right)=B_{m}^{m} u_{m}^{k}+\sum_{j=0}^{m-1} B_{j}^{m} \mathfrak{u}_{j}^{k}
$$

we have

$$
\begin{aligned}
& \mathcal{U}_{m}^{k}=\left(B_{m}^{m}\right)^{-1} \sum_{j=0}^{m-1}\left(\alpha_{m}^{s, d} B_{j}^{m+1}+\beta_{m}^{s, d} B_{j}^{m}+\gamma_{m}^{s, d} B_{j}^{m-1}\right) \mathfrak{U}_{j}^{k-1} \\
& +\left(B_{m}^{m}\right)^{-1}\left(\left(\alpha_{m}^{s, d} B_{m}^{m+1}+\beta_{m}^{s, d} B_{m}^{m}\right) \mathcal{U}_{m}^{k-1}+\alpha_{m}^{s, d} B_{m+1}^{m+1} \mathcal{u}_{m+1}^{k-1}-\sum_{j=0}^{m-1} B_{j}^{m} \mathfrak{u}_{j}^{k}\right) .
\end{aligned}
$$


For $m=k$ we have

$$
\mathcal{U}\left(\left(x^{s}\right)^{k} \mathcal{B}_{k}\right)=\gamma_{k}^{s, d} \gamma_{k-1}^{s, d} \cdots \gamma_{1}^{s, d} B_{0}^{0} \mathcal{U}_{0}^{0},
$$

and so,

$$
\mathcal{U}_{k}^{k}=\left(B_{k}^{k}\right)^{-1}\left(\gamma_{k}^{s, d} \gamma_{k-1}^{s, d} \cdots \gamma_{1}^{s, d} B_{0}^{0} \mathcal{U}_{0}^{0}-\sum_{j=0}^{k-1} B_{j}^{k} \mathcal{U}_{j}^{k}\right) .
$$

For $m>k$ we have $\mathcal{U}\left(\left(x^{s}\right)^{k} \mathcal{B}_{m}\right)=0_{s d \times s d}$, i.e.

$$
\mathfrak{U}_{m}^{k}=-\sum_{j=0}^{m-1}\left(B_{m}^{m}\right)^{-1} B_{j}^{m} \mathcal{U}_{j}^{k} .
$$

Therefore, the moments associated to the vector of linear functionals $\mathcal{U}$ are uniquely determined from (11). Hence, the result is proved.

Notice that, in matrix notation the three-term recurrence relation of the previous Theorem, (12), is written by

$$
J\left[\begin{array}{c}
\mathcal{B}_{0} \\
\vdots \\
\mathcal{B}_{m} \\
\vdots
\end{array}\right]=x^{s}\left[\begin{array}{c}
\mathcal{B}_{0} \\
\vdots \\
\mathcal{B}_{m} \\
\vdots
\end{array}\right]
$$

where the tridiagonal matrix by blocks

$$
J=\left[\begin{array}{cccccc}
\beta_{0}^{s, d} & \alpha_{0}^{s, d} & 0_{s d \times s d} & & & \\
\gamma_{1}^{s, d} & \beta_{1}^{s, d} & \alpha_{1}^{s, d} & 0_{s d \times s d} & & \\
0_{s d \times s d} & \gamma_{2}^{s, d} & \beta_{2}^{s, d} & \alpha_{2}^{s, d} & 0_{s d \times s d} & \\
& \ddots & \ddots & \ddots & \ddots & \ddots
\end{array}\right],
$$

is called block Jacobi matrix associated to the vector of functionals $\mathcal{U}$, and the uniquely determined vector polynomial sequence $\left\{\mathcal{B}_{m}\right\}$ associated to the vector of functionals $\mathcal{U}$, is called type II multiple vector orthogonal sequence with respect to the vector of functionals $\mathcal{U}$.

\section{Type II Hermite-PadÉ approximation}

Definition 7. Let $\mathcal{U}=\left[\begin{array}{lll}v^{1} & \cdots & v^{s d}\end{array}\right]$ be a vector of linear functionals. We define the matrix generating function associated to $\mathcal{U}, \mathcal{F}$, by

$$
\mathcal{F}(z):=\mathcal{U}_{x}\left(\frac{\mathcal{P}_{0}(x)}{z-x^{s}}\right)=\left[\begin{array}{ccc}
v_{x}^{1}\left(\frac{1}{z-x^{s}}\right) & \cdots & v_{x}^{s d}\left(\frac{1}{z-x^{s}}\right) \\
\vdots & \ddots & \vdots \\
v_{x}^{1}\left(\frac{x^{s d-1}}{z-x^{s}}\right) & \cdots & v_{x}^{s d}\left(\frac{x^{s d-1}}{z-x^{s}}\right)
\end{array}\right] .
$$


Being,

$$
\frac{1}{z-x^{s}}=\frac{1}{z} \sum_{k=0}^{\infty}\left(\frac{x^{s}}{z}\right)^{k} \text { for }\left|x^{s}\right|<|z|
$$

we have $\mathcal{F}(z)=\sum_{k=0}^{\infty} \frac{\left(\left(x^{s}\right)^{k} \mathcal{U}_{x}\right)\left(\mathcal{P}_{0}(x)\right)}{z^{k+1}}$.

Theorem 7. Let $\mathcal{U}=\left[\begin{array}{lll}v^{1} & \cdots & v^{s d}\end{array}\right]$ be a regular vector of linear functionals, $\left\{\mathcal{B}_{m}\right\}$ the vector type II multiple orthogonal polynomials sequence with respect to $\mathcal{U}, J$ the block Jacobi matrix associated, given in (14) and $\mathcal{R}$ the resolvent function associated, i.e.

$$
\mathcal{R}(z)=\sum_{n=0}^{\infty} \frac{e_{0}^{t} J^{n} e_{0}}{z^{n+1}}, \text { where } e_{0}=\left[I_{s d \times s d} 0_{s d \times s d} \cdots\right]^{T} .
$$

Then, $\mathcal{R}(z)=B_{0}^{0} \mathcal{F}(z)\left(\mathcal{U}\left(\mathcal{P}_{0}\right)\right)^{-1}\left(B_{0}^{0}\right)^{-1}$, where $B_{0}^{0}$ is the matrix coefficient in $\mathcal{B}_{0}=B_{0}^{0} \mathcal{P}_{0}$.

Proof. In order to determine the value of $e_{0}^{t} J^{n} e_{0}, n \in \mathbb{N}$, we consider the matrix identity (13), from which we can obtain,

$$
J^{n}\left[\begin{array}{c}
\mathcal{B}_{0}(x) \\
\vdots \\
\mathcal{B}_{m}(x) \\
\vdots
\end{array}\right]=\left(x^{s}\right)^{n}\left[\begin{array}{c}
\mathcal{B}_{0}(x) \\
\vdots \\
\mathcal{B}_{m}(x) \\
\vdots
\end{array}\right], n \in \mathbb{N} .
$$

Let $\left(x^{s}\right)^{n} \mathcal{B}_{0}(x)=\sum_{j=0}^{n} \eta_{j, n}^{0} \mathcal{B}_{j}(x)$.

By (17), $e_{0}^{t} J^{n} e_{0}, n \in \mathbb{N}$, is given by $\eta_{0, n}^{0}$. Applying the vector of linear functionals $\mathcal{U}$ to both members of the previous matrix identity, we have

$$
\eta_{0, n}^{0}=\left(\left(x^{s}\right)^{n} \mathcal{U}\right)\left(\mathcal{B}_{0}\right)\left(\mathcal{U}\left(\mathcal{B}_{0}\right)\right)^{-1}
$$

Using $\mathcal{B}_{0}=B_{0}^{0} \mathcal{P}_{0}$, we have

$$
\eta_{0, n}^{0}=B_{0}^{0}\left(\left(x^{s}\right)^{n} \mathcal{U}\right)\left(\mathcal{P}_{0}\right)\left(\mathcal{U}\left(\mathcal{P}_{0}\right)\right)^{-1}\left(B_{0}^{0}\right)^{-1} .
$$

Hence,

$$
\mathcal{R}(z)=B_{0}^{0}\left\{\sum_{n=0}^{\infty} \frac{\left(\left(x^{s}\right)^{n} \mathcal{U}\right)\left(\mathcal{P}_{0}\right)\left(\mathcal{U}\left(\mathcal{P}_{0}\right)\right)^{-1}}{z^{n+1}}\right\}\left(B_{0}^{0}\right)^{-1},
$$

as we wanted to prove. 
The vector sequence of polynomials $\left\{\mathcal{B}_{m}\right\}$, where

$$
\mathcal{B}_{m}=\left[\begin{array}{lll}
B_{m s d} & \cdots & B_{(m+1) s d-1}
\end{array}\right]^{T}, m \in \mathbb{N}
$$

and $B_{n}$ is a monic polynomial of degree $n$ can be written as

$$
\mathcal{B}_{n}=\sum_{j=0}^{n} B_{j}^{n} \mathcal{P}_{j}, \quad B_{j}^{n} \in \mathcal{M}_{s d \times s d},
$$

where the matrix coefficients $B_{j}^{n}, j=0,1, \ldots, n$ are uniquely determined and $B_{n}^{n}$ is a regular lower triangular matrix.

Taking into account (6) we have that $\mathcal{P}_{j}=\left(x^{s d}\right)^{j} \mathcal{P}_{0}, j \in \mathbb{N}$. Therefore, $\mathcal{B}_{n}=V_{n}\left(x^{s d}\right) \mathcal{P}_{0}$, where $V_{n}$ is a matrix polynomial of degree $n$ and dimension $s d$, given by $V_{n}(x)=\sum_{j=0}^{n} B_{j}^{n} x^{j}, \quad B_{j}^{n} \in \mathcal{M}_{s d \times s d}$. Now, we present a reinterpretation of type II Hermite-Padé approximation in terms of the matrix functions.

Definition 8. Let $\left\{\mathcal{B}_{m}\right\}$ be a vector sequence of polynomials and $\mathcal{U}$ a vector of linear functionals. To the sequence of polynomials $\left\{\mathcal{B}_{m-1}^{(1)}\right\}$ given by

$$
\mathcal{B}_{m-1}^{(1)}(z):=\mathcal{U}_{x}\left(\frac{V_{m}\left(z^{d}\right)-V_{m}\left(x^{s d}\right)}{z-x^{s}} \mathcal{P}_{0}(x)\right),
$$

where $\mathcal{U}_{x}$ represents the action of $\mathcal{U}$ over the variable $x$, and $\mathcal{B}_{n}=$ $V_{n}\left(x^{s d}\right) \mathcal{P}_{0}$, we designate sequence of polynomials associated to $\left\{\mathcal{B}_{m}\right\}$ and to $\mathcal{U}$.

Theorem 8. Let $\mathcal{U}$ be a regular vector of linear functionals, $\left\{\mathcal{B}_{m}\right\}$ a vector sequence of polynomials, $\mathcal{B}_{n}=V_{n}\left(x^{s d}\right) \mathcal{P}_{0}$, where $V_{n}(x)=$ $\sum_{j=0}^{n} B_{j}^{n} x^{j}, B_{j}^{n} \in \mathcal{M}_{s d \times s d}, B_{n}^{n}$ is a regular lower triangular matrix with entries equal to 1 in the diagonal. $\left\{\mathcal{B}_{m-1}^{(1)}\right\}$ the sequence of associated polynomials and $\mathcal{F}$ the matrix generating function defined in (15). Then $\left\{\mathcal{B}_{m}\right\}$ is the vector type II multiple orthogonal with respect to the vector of linear functionals $\mathcal{U}$ if, and only if,

$$
V_{m}\left(z^{d}\right) \mathcal{F}(z)-\mathcal{B}_{m-1}^{(1)}(z)=\sum_{k=m}^{\infty} \frac{\left(\left(x^{s}\right)^{k} \mathcal{U}_{x}\right)\left(\mathcal{B}_{m}(x)\right)}{z^{k+1}} .
$$

and $\left(\left(x^{s}\right)^{k} \mathcal{U}_{x}\right)\left(\mathcal{B}_{m}(x)\right)=\Delta_{m}$, where $\Delta_{m}$ is a regular upper triangular matrix.

Proof. Taking into account the Definition 8, we have

$$
\begin{aligned}
\mathcal{B}_{m-1}^{(1)}(z) & =\mathcal{U}_{x}\left(\frac{V_{m}\left(z^{d}\right)-V_{m}\left(x^{s d}\right)}{z-x^{s}} \mathcal{P}_{0}(x)\right) \\
& =V_{m}\left(z^{d}\right) \mathcal{F}(z)-\mathcal{U}_{x}\left(\frac{V_{m}\left(x^{s d}\right)}{z-x^{s}} \mathcal{P}_{0}(x)\right)
\end{aligned}
$$


i.e. $V_{m}\left(z^{d}\right) \mathcal{F}(z)-\mathcal{B}_{m-1}^{(1)}(z)=\mathcal{U}_{x}\left(\frac{V_{m}\left(x^{s d}\right)}{z-x^{s}} \mathcal{P}_{0}(x)\right)$.

Taking into account (16) we have

$$
V_{m}\left(z^{d}\right) \mathcal{F}(z)-\mathcal{B}_{m-1}^{(1)}(z)=\sum_{k=0}^{\infty} \frac{\left(\left(x^{s}\right)^{k} \mathcal{U}_{x}\right)\left(\mathcal{B}_{m}(x)\right)}{z^{k+1}} .
$$

Hence, we get the desired result.

\section{ACKNOWLEDGMENT}

We want to thank the referees for the suggestions and references that improved the manuscript. We also want to thank Eduardo Godoy for the help in generating the example given.

\section{REFERENCES}

[1] A.I. Aptekarev, Multiple orthogonal polynomials, J. Comput. Appl. Math. 99 (1998) 423-447.

[2] A.I. Aptekarev, A. Branquinho and W. Van Assche, Multiple orthogonal polynomials for classical weights, Trans. Amer. Math. Soc. 335 (2003) 3887-3914.

[3] J. Arvesú, Coussement and W. Van Assche, Some discrete multiple orthogonal polynomials, J. Comput. Appl. Math. 153 (2003) no. 1-2, 19-45.

[4] D. Barrios Rolanía, A. Branquinho, and A. Foulquié Moreno, Dynamics and interpretation of some integrable systems via multiple orthogonal polynomials, J. Math. Anal. Appl. 361 (2010), no. 2, 358-370.

[5] B. Beckermann, A reliable method for computing $M$-Padé approximants on arbitrary staircases, J. Comput. Appl. Math. 40 (1992), no. 1, 19-42.

[6] B. Beckermann, J. Gilewicz, and V. Kaliaguine, On the definition and normality of a general table of simultaneous Padé approximants, J. Approx. Theory $\mathbf{7 7}$ (1994), no. 1, 65-73.

[7] B. Beckermann and G. Labahn, A uniform approach for Hermite Padé and simultaneous Padé approximants and their matrix-type generalizations, Numer. Algorithms 3 (1992), no. 1-4, 45-54.

[8] A. Bultheel, Recursive algorithms for the matrix Padé problem, Math. Comp. 35 (1980), no. 151, 875-892.

[9] A. Bultheel, Recursive relations for block Hankel and Toeplitz systems. I. Direct recursions, J. Comput. Appl. Math. 10 (1984), no. 3, 301-328.

[10] A. Bultheel, Recursive relations for block Hankel and Toeplitz systems. II. Dual recursions, J. Comput. Appl. Math. 10 (1984), no. 3, 329-354.

[11] S.K. Burley, S.O. John, and J. Nuttall, Vector orthogonal polynomials, SIAM J. Numer. Anal. 18 (1981), no. 5, 919-924.

[12] J. Bustamante and G. Lopes Lagomasino, Hermite-Padé approximations for Nikishin systems of analytic functions, Russian Acad. Sci. Sb. Math. 77 (1994), no. 2, 367-384.

[13] J. Coussement and W. Van Assche, Differential equations for multiple orthogonal polynomials with respect to classical weights: raising and lowering operators, J. Phys. A 39 (2006) no. 13, 3311-3318. 
[14] K. Douak and P. Maroni, Une caractérisation des polynômes d-orthogonaux classiques, J. Approx. Th. 82 (1995) 177-204.

[15] A.J. Durán, A generalization of Favard's theorem for polynomials satisfying a recurrence relation, J. Approx. Th. 74 (1993) 83-109.

[16] W.D. Evans, L.L. Littlejohn and F. Marcellán, On recurrence relations for Sobolev orthogonal polynomials, SIAM J. Math. Anal. 26 (1995) 446-467.

[17] M.E.H. Ismail, Classical and quantum orthogonal polynomials in one variable, Encyclopedia of Mathematics and its Applications 98, Cambridge University Press, 2005.

[18] V. Kaliaguine, The operator moment problem, vector continued fractions and an explicit form of the Favard theorem for vector orthogonal polynomials, J. Comput. Appl. Math. 65 (1995) no. 1-3, 181-193.

[19] D.W. Lee, Difference equations for discrete classical multiple orthogonal polynomials, J. Approx. Th. 150 (2008) no. 2, 132-152.

[20] P. Maroni, Two-dimensional orthogonal polynomials, their associated sets and the co-recursive sets, Numer. Algorithms 3 (1992) 299-312.

[21] P. Maroni, Orthogonality and recurrences of polynomials of order greater than two, Ann. Fac. Sci. Toulouse Math. 10 (1989), no. 1, 105-139.

[22] E.M. Nikishin and V.N. Sorokin, Rational Approximations and Orthogonality, Transl. Math. Monographs, 92, Amer. Math. Soc. Providence RI, 1991.

[23] V.N. Sorokin and J. Van Iseghem, Algebraic aspects of matrix orthogonality for vector polynomials, J. Approx. Theory 90 (1997), 97-116.

[24] W. Van Assche, Analytic number theory and approximation, Coimbra Lecture Notes on Orthogonal Polynomials (A. Branquinho and A.P. Foulquié Moreno, eds.), Nova Science Publishers, 2007, 197-229.

[25] W. Van Assche and E. Coussement, Some classical multiple orthogonal polynomials, J. Comput. Appl. Math. 127 (2001), 317-347.

[26] M. Van Barel and A. Bultheel, A general module-theoretic framework for vector M-Padé and matrix rational interpolation, Numer. Algorithms 3 (1992), no. 1-4, 451-461.

[27] J. Van Iseghem, Vector orthogonal relations. Vector QD-algorithm, J. Comput. Appl. Math. 19 (1987), 141-150.

CMUC, Department of Mathematics, University of Coimbra, Largo

D. Dinis, 3001-454 Coimbra, Portugal

E-mail address: ajplb@mat.uc.pt

School of Technology and Management, Polytechnic Institute of Leiria, Campus 2 - Morro do Lena - Alto do Vieiro, 2411 - 901 Leiria PorTugal

E-mail address: lmsc@estg.ipleiria.pt

Ui Matemática e AplicaÇões from University of Aveiro, Departamento de Matemática, Universidade de Aveiro, Campus de Santiago 3810, Aveiro, Portugal

E-mail address: foulquie@ua.pt 